\title{
Development and Validation of the Multidimensional Library Anxiety Scale
}

\section{Doris J. Van Kampen}

This article reports on the development and validation of the Multidimensional Library Anxiety Scale, which was designed to assess six dimensions of an individual's perception of an academic library and the information search process (ISP). A two-part study was undertaken for the development of the instrument. In part one, twenty-one participants completed a pilot questionnaire that assisted the researcher in developing the instrument. In part two, 278 participants completed a revised questionnaire consisting of a 54-item Likert-type scale that assessed levels of library anxiety experienced by students enrolled in a doctoral degree-granting program at an urban southeastern university.

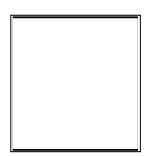

rustration over how to begin a search for a topic and how to find information related to the topic are recurrent themes when students write about their library experiences. ${ }^{1-3}$ Library anxiety has been reported as a characteristic among graduate and undergraduate students, with an estimated 95 percent of college students engaging in frequent academic procrastination because of library anxiety. ${ }^{4,5}$ This phenomenon has been discussed in the literature and quantitatively measured by a number of researchers over the past decade using the Library Anxiety Scale (LAS) developed by Sharon Bostick. The Library Anxiety Scale was created "to categorize and measure this concept in college students." 6

The first formalized study of library anxiety as a phenomenon occurred in the mid-1980s, when Constance Mellon conducted a two-year study of undergraduate beginning composition classes at a southern university. ${ }^{7}$ Twenty English instructors assigned and collected personal writings from students describing their library search experiences. The diary-like entries described how students undertook their searches and their feelings about the process. The major themes that emerged were confusion, fear, and the feeling of being lost. Four strands concerning the "lost in the library" theme were noted: "the size of the library; not knowing where things were; not knowing what to do; and not knowing how to begin the [library] research process." 8 From her research, Mellon developed a theory about library anxiety, which stated, "When confronted with the need to gather information in the library ... many students become so anxious that they are unable to approach the problem logically or effectively." 9

Mellon's theory of library anxiety was qualitatively tested, but the question remained as to whether a valid and reliable 
instrument could be developed to quantitatively measure library anxiety. In 1992, Sharon Bostick developed and validated the LAS, which was designed to categorize and measure library anxiety in college students at two- and four- year institutions. ${ }^{10}$ The LAS measures five components: (1) barriers with staff, (2) affective barriers, (3) comfort with the library, (4) knowledge of the library, and (5) mechanical barriers. "Barriers with staff" refers to a library patron's perception of library staff as intimidating and unapproachable as well as being too busy to help. "Affective barriers" measures the respondent's feeling of adequacy when using the library, and "comfort" reflects how safe, welcoming, and nonthreatening the library is perceived to be. Feelings of inadequacy and discomfort regarding the library have been acknowledged as a limiting factor to students' research efforts in the library. "Knowledge of the library" reflects student perspectives on how familiar they think they are with the library and its resources, and "mechanical barriers" examines the feelings that emerge as a result of student reliance on library equipment.

However, when the LAS was developed, scant information and few theories were available on the user's feelings during the research process itself; the Internet was not yet widely used as a research tool, and database access was limited primarily to the physical confines of the library. User studies were limited mostly to statistics reflecting usage and how the user interacted with the library as a system as opposed to examining user perspectives of the research process. Even now, the majority of current user research focuses on levels of satisfaction with services or on how patrons interact with the library's database or Web site, rather than on how a person may feel about the library, technology, and the process of searching for information. "Most of the current patron user research in academic libraries focuses on levels of satisfaction with current services and on how patrons are navigating the library OPAC or website." ${ }^{11}$ A 2001 sampling of reports by this author from user satisfaction surveys in library research studies did not find any that indicated that the library in question was determined to be unsatisfactory in any significant area. The vast majority of surveys rated library satisfaction levels as high or very high. However, many of the survey results noted an increased demand for more full-text databases and online services.

The researcher was interested in whether doctoral students, who should have had experience with the ISP and using the library, showed evidence of library anxiety, and if they did, whether anxiety levels varied over time. Other questions of interest to the researcher concerned how gender might influence use of traditional or online use of resources and whether doctoral students who exhibited higher levels of anxiety stated a preference for using online resources. However, before these questions could be answered, an instrument needed to be located or developed for this purpose. Because of the age of the original LAS and other developments in the field of library user research, it was deemed appropriate to create a new instrument based on the LAS, named the Multidimensional Library Anxiety Scale (MLAS), which would take these factors and the researcher's questions into consideration. ${ }^{12}$ Although this instrument in no way invalidates the LAS, it does take into consideration off-campus use of library resources and doctoral students' attitudes during the course of their dissertations. Statements regarding respondents' preferences concerning use of the library online or in person and statements that reflected comfort level with computers were included.

The initial set of potential statements concerning online resources was e-mailed or mailed using the U. S. postal service with a cover letter to librarians and faculty from academic libraries and to Sharon Bostick. Codes were developed for use in SPSS 10.0 using Bostick's LAS as a reference tool for placement of the variable within a general classification 
scheme. Following revisions to the survey, a pilot study was conducted to determine the potential validity of the instrument.

The pilot study instrument consisted of fifty-seven statements and was conducted during the summer semester of 2001 at a large metropolitan-based university in the South. The sample population consisted of eighteen doctoral students enrolled in EDG 7931, Structural Equation Models, an elective course for all graduate education majors in the College of Education. The sample population selected was based on willingness of faculty members to allow students to have time in class to participate.

A five point Likert-type scale was used for the pilot survey, with available answers being "strongly agree," "agree," "undecided," "disagree," and "strongly disagree." The pilot instrument statements were printed on a word processor and photocopied. Directions were printed at the top, and a business card was included in the packet inviting the students to e-mail comments or questions about the survey to the researcher.

A test-retest method was used for the pilot to improve reliability of the instrument; this method is used when a researcher is interested in whether the participants will answer a set of statements the same way more than once over a period of time. The pilot study participants were given the survey first with coded envelopes to identify respondents and again three weeks later to determine the consistency of their answers. The directions asked students to respond to the statements by circling the number that best correlated with their feelings regarding the statement.

Upon completion of the pilot study, data were input into SPSS 10.0. Negatively worded statements were reverse-scored so that all the statements were scored in the same direction. Exploratory Factor Analysis (EFA) was utilized on the data collected to extract latent variables and examine correlations. According to Kevin Kieffer, "EFA is an analytic technique in which the pri- mary concern is to reduce a larger set of variables into a smaller and more manageable set based in the consistency of the data." ${ }^{\prime 3}$ EFA assists with detecting and analyzing possible patterns based on the correlations among the variables. It is used when there is "initial uncertainty as to the number of factors being measured."14 The purpose was to summarize the interrelationships among the fifty-three variables (statements) in order to assist in the analysis and conceptualization of possible categories. The extraction method used was principle components, with a varimax procedure, excluding cases listwise and sorting factors by size. Using this method, variables with an absolute value of less than .30 were excluded. The reason for excluding variables below this threshold was to eliminate variables with lower correlations and to retain variables with higher correlations, aiding in the interpretation of the results. If one examines similar research using EFA, one will find that most studies use .30 as a rule of thumb for determining "noteworthy coefficient magnitudes."15

The initial factor analysis yielded eleven factors in twenty-three iterations. For the pilot study, a bivariate correlation analysis using Pearson's $r$ was completed after breaking each factor into clusters, and then the statements and the correlations were examined. The researcher reflected on the software program's placement and decided to move several items from one factor to another until most items were correlating at .45 or higher. This was done based on consultation with an expert in the field and on the researcher's own opinion as to where the items would make the best fit because "analytic results can inform the definitions we wish to create."16 Correlations were rerun on each factor after the items were moved to examine the interaction among the items. Most correlations were significant at the .01 level, and all but a few items were significant at the .05 level. Because of the small size of the pilot sample, it was decided to keep several of the items with correlations more than .30 and less than .45 to determine whether the correlations were due more 
to the sample's size and less to the item itself. Revisions to the instrument were based the following criteria:

- lack of correlation with other items (item removed);

- perceived lack of clarity based on participant feedback (item rewritten);

- relocation within the instrument to reflect intercorrelations;

- perception of respondents (based on how they answered) that the item appeared to measure some other construct than the one the researcher had believed it would.

Each variable retained the same code that had been assigned during the pilot study. The researcher decided that it would be better to retain the same codes for each statement rather than create new ones so that it would be easier to refer back to the pilot data, if needed.

\section{Data Collection: Survey}

The full study was conducted by mailing questionnaires to the entire doctoral population of 554 who had been enrolled at the institution during the spring of 2001. The questionnaires were mailed from January 1 through February 23, 2002. A letter explaining the purpose of the survey was sent with the instrument. Each letter was personalized and signed by hand, with one statement in red ink to emphasize the need for each student's participation. All envelopes were handwritten, with a collector's stamp on each envelop and a self-addressed stamped envelope included with each mailing. A bookmark was enclosed with each survey as a token gift. Potential respondents were informed that if they returned a completed survey by January 21, 2002, they would be eligible for several small prizes. A follow-up postcard was mailed a little over two weeks after the initial mailing. Sending a duplicate packet was considered but because of the cost of each packet and the diminishing return rate, the researcher decided not to resend the packets. It was estimated that a high percentage (over 50\%) would return the survey due to the implementation of several strategies, which have been reported to increase response rates for mail surveys. ${ }^{17-}$ 19 The cost for printing and mailing the initial packet with four first-class stamps, two envelopes, five sheets of paper, and the bookmark was $\$ 2.10$ per mailing, for a total of $\$ 1,163.40$. The cost of purchasing, printing, and mailing the postcard reminders was 49 cents per postcard (\$131.32 total), with 268 postcards being mailed on January 19, 2002. After ten weeks, a total of 278 surveys had been completed and returned, bringing the cost rate to $\$ 4.65$ per survey.

Data from survey responses were input into SPSS 10.0. Negatively worded variables were reverse-scored. Measures of central tendency and dispersion were explored after a new factor was created using SPSS 10.0 Syntax COMPUTE [ ] command, which compiled the total score for each case, excluding cases with missing data. Distribution of the data was symmetric, as depicted in the stem \& leaf plot. A stem \& leaf plot is a frequency table that has been graphically depicted, similar to a bar graph rotated clockwise. (See figure 1.)

EFA was utilized on the survey data to extract latent variables and examine corre-

\begin{tabular}{c} 
FIGURE 1 \\
Stem \& Leaf Plot \\
\hline \hline
\end{tabular}

\section{Frequency Stem \& Leaf}

\begin{tabular}{rl}
\hline 2.00 & $16 . \&$ \\
4.00 & $17 . \& \&$ \\
16.00 & $18.134579 \&$ \\
17.00 & $19.13389 \& \&$ \\
22.00 & $20.02345568 \&$ \\
38.00 & $21.022334455677788999 \&$ \\
45.00 & 22.00122344456677888999 \\
41.00 & $23.000134445556677899 \&$ \\
33.00 & $24.1111235567789 \&$ \\
23.00 & $25.023344557 \&$ \\
6.00 & $26.04 \&$ \\
1.00 & $27 . \&$
\end{tabular}

Stem width: 10.00. Each leaf: 2 case(s) \& denotes fractional leaves 
lations. The extraction method used was principal components, rotated orthagonally using a varimax procedure. "Principle components are very closely tied to the original variables, with each subject's score on a principle component being a linear combination of his or her scores on the original variables." 20

Factors with an absolute value of less than .30 were excluded. This was done based on the researcher's readings in this field and after discussion with an expert in the field on where an appropriate cut-off point

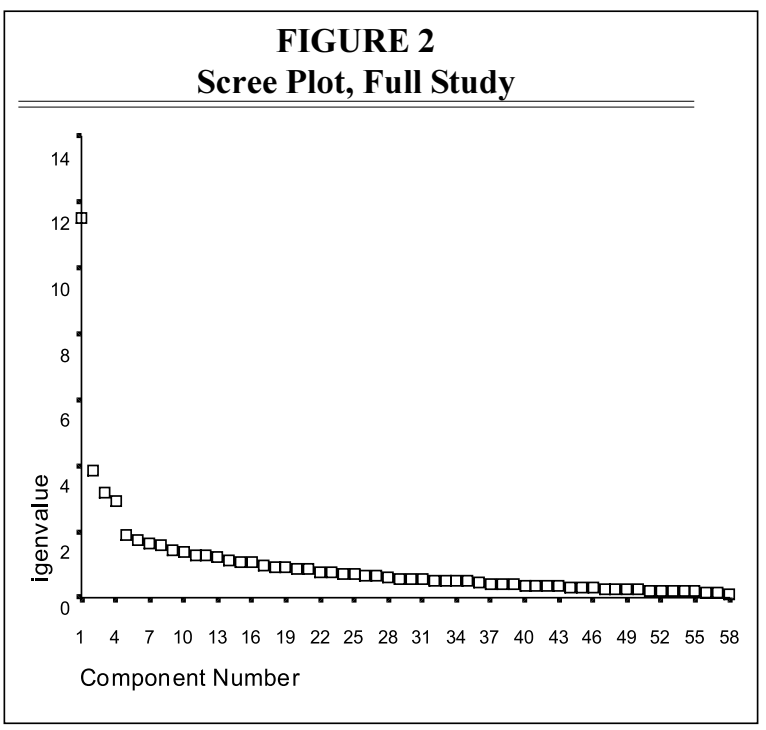
should be located. The initial factor analysis yielded sixteen components after thirty-one iterations. The Scree plot leveled off after five factors. (See figure 2.)

After examining the Scree plot, eigenvalues, components matrix, and correlations matrix, the researcher decided to force the factor analysis into seven components, as all but one of the components factoring on factors 8-16 were also factoring on components $1-7$. The seven factors fell generally into the following categories:

1. perceived knowledge of the library and confidence concerning the ISP;

2. the ISP and general library anxiety;

3. perceived importance of the library and constraints to use;

4. comfort level with technology, interaction with staff;

5. library independence;

6. comfort level while inside the library building;

7. perceived barriers concerning staff.

Upon further examination of the factors, the researcher decided factors seven and four had too many overlapping variables (staff issues) to be considered as separate factors and forced the factor analysis into six factors to avoid attenuating the results. Each component retained had at least three significant loadings and an eigenvalue greater than 1.0.

Each retained component was considered a major component and, as such, would probably be of interest to other investigators. ${ }^{21}$ Once again, the extraction method used was principal components.

Table 1 represents the initial eigenvalues of the six factors, the rotated (trace) eigenvalues for each factor, the percent of variance and cumulative variance, and the number of items factoring on each component as derived from the forced factor analysis.

To test for internal consistency, Cronbach's Alpha was computed on the fifty-three statements. Cronbach's Alpha is a common index of reliability. When using this measure of reliability, the closer the results are to 1.0, the higher the estimate of reliability. The resultant alpha coefficient of .88 for all fifty-three items provided evidence of adequate internal consistency.

A new factor was derived for each of the six components using the COMPUTE factor [ ] syntax command in SPSS 10.0. Keep in mind that a factor is not a variable; it is "the reduction of a larger set of variables into a smaller and more manageable set based on the consistency of the data. ${ }^{\prime 22} \mathrm{Fac}-$ tors were labeled as follows:

1. KNOW: Comfort and confidence (library independence) when using the library; 
2. ISPLIB: The Information Search Process and general Library Anxiety;

3. STAF: Perceived barriers concerning staff;

4. IMPLIB: Perceived importance of understanding how to use the library;

5. TECH: Comfort level with technology and as it applies to the library;

6. BUIL: Comfort level while inside the library building.

Labels developed in this manner were designed to correspond with the forced factor analysis. The rotated components matrix was examined for overlapping variables, and some overlapping variables were removed from one or more of the factors for better analysis. The decision as to which items to remove was made after examining the rotated components matrix, the correlations matrix, descriptive statistics within Cronbach's Alpha for each factor's item, scale, and scale, if item removed. Along with overlapping variables on some factors, two variables did not manifest on any factor. If an overlapping variable was considered of interest to the researcher's questions in more than one area, a judgment was made as to whether to leave it on two factors. No variable was allowed to overlap on more than two factors.

Cronbach's Alpha was then run on each separate factor to test for internal consistency within each factor. Score reliability, as measured by coefficient alpha, for each subscale was as follows:

1. Comfort and confidence when using the library $=.86$

2. Information Search Process and general Library Anxiety $=.87$

3. Barriers concerning staff $=.73$

4. Importance of understanding how to use the library $=.79$

5. Comfort level with technology and as it applies to the library $=.73$

6. Comfort level while inside the library building $=.74$

Although the average interitem correlation was high, several items were removed after Cronbach's Alpha had been run. This is considered a normal procedure. Some items were removed in view of the fact that, although they were giving adequate correlations and did not detract from the Alpha scores, they were not enhancing the scores and overlapped with another factor. One item (would rather use the library in person) on factor 5 was removed from factor 5 , but not from the scale when it was noted that it was depressing the Alpha score of that factor by .18 (before removal: .5513; after removal: .7322). This was done because there was insufficient evidence that the item was measuring the same underlying construct as the other items within that factor. This item would perhaps show a different correlation with regard to the other factors if the survey were given to another population.

The following conclusions were drawn concerning the survey instrument:

- Library anxiety in an academic library setting can be measured using this survey instrument.

- Intercorrelations for all fifty-three factors were sufficient to ensure internal consistency among the items.

- Intercorrelations for each factor were sufficient to ensure internal consistency among the items within each factor.

- If used on a similar population, the instrument should be sufficiently stable 
to produce results that measure the six dimensions of library anxiety identified during factor analysis.

The first phase of research, developing an instrument, was considered complete. The survey had been returned by a sufficient number of students, the factor analysis did not reveal any significant problems, and further analysis could begin.

\section{Summary}

This developmental study of the MLAS indicated that the questionnaire scale showed good internal consistency and construct validity and that the scale has the potential to be a useful tool for deter- mining what aspects of the library and the information search process are perceived to be barriers by graduate students. Researchers interested in graduate students as subjects might wish to use the scale, particularly with master's- and doctoral-level subjects involved in a thesis or dissertation. Further publication by the author concerning the actual results of the study are forthcoming. Researchers interested in obtaining a copy of the scale are encouraged to contact the author. Additional research into the usefulness and stability of the scale for similar or dissimilar populations at other institutions is indicated.

\section{Notes}

1. Constance Mellon, "Library Anxiety: A Grounded Theory and Its Development," College $\mathcal{E}$ Research Libraries 47 (Mar. 1986): 160-65.

2. —- Naturalistic Inquiry for Library Science: Methods and Applications for Research, Evaluation, and Teaching (New York: Greenwood Pr., 1990).

3. Carol C. Kuhlthau, Seeking Meaning: A Process Approach to Library and Information Services (Norwood, N.J.: Ablex, 1993).

4. Sharon Bostick, "The Development and Validation of a Library Anxiety Scale" (Detroit, Mich.: Wayne State University, 1992). Abstract in Digital Dissertation.

5. Anthony Onwuegbuzie and Qun Jiao, "I'll Go to the Library Later: The Relationship between Academic Procrastination and Library Anxiety," College \& Research Libraries 61(1): 45-54. Available online from http://library.ucf.edu. (Retrieved 1 October 2001.)

6. Bostick, "The Development and Validation of a Library Anxiety Scale."

7. Mellon, "Attitudes: The Forgotten Dimension in Library Instruction," Library Journal 113 (Sept. 1988): 137-39.

8. Ibid.

9. Ibid

10. Bostick, "The Development and Validation of a Library Anxiety Scale."

11. Doris J. Van Kampen, Library Anxiety, the Information Search Process, and Doctoral Students' Use of the Library (Orlando, Fla.: University of Central Florida, 2003).

12. MLAS @ 2003 Doris J. Van Kampen and Sharon Bostick. To obtain a copy of the MLAS, please contact the author at doris.vankampen@saintleo.edu.

13. Kevin Kieffer, "An Introductory Primer on the Appropriate Use of Exploratory and Confirmatory Factor Analysis," Research in Schools 6 (fall 1999): 75-92.

14. Joseph Capelleri and Robert Gerber, Encyclopedia of Biopharmaceutical Statistics (Cambridge, Mass.: Cambridge Biostatistics and Data Management, Inc., 2003).

15. Kieffer, "An Introductory Primer on the Appropriate Use of Exploratory and Confirmatory Factor Analysis," 80.

16. Bruce Thompson and Larry Daniel, "Factor Analytic Evidence for the Construct Validity of Scores: A Historical Overview and Some Guidelines," Educational and Psychological Measurement 56 (Apr. 1996): 197-208.

17. Earl R. Babbie, Survey Research Methods (Belmont, Calif.: Wadsworth, 1990).

18. Francis Buttle, "Questionnaire Color and Mail Survey Response Rate," Journal of the Market Research Society 39 (Oct. 1997): 625-26.

19. David Dodd and Barbara Markwiese, "Survey Response Rate as a Function of Personalized Signature on Cover Letter," Journal of Social Psychology 127 (Feb. 1987): 97-98.

20. Richard Harris, Primer of Multivariate Statistics (London: Lawrence Erlbaum Assoc., 2001).

21. William Zwick and Wayne Velicer, "Comparison of Five Rules for Determining the Number of Components to Retain," Psychological Bulletin 199 (1986): 432-42.

22. Kieffer "An Introductory Primer on the Appropriate Use of Exploratory and Confirmatory Factor Analysis," 76. 